\title{
Efficient isolation of sperm with high DNA integrity and stable chromatin packaging by a combination of density-gradient centrifugation and magnetic- activated cell sorting
}

\author{
Hee-Jun Chi, Su-Jin Kwak, Seok-Gi Kim, Youn-Young Kim, Ji-Young Park, Chang-Seok Yoo, Il-Hae Park, Hong-Gil Sun, \\ Jae-Won Kim, Kyeong-Ho Lee
}

IVF Center, Mamapapa and Baby Clinic, Ulsan, Korea

Objective: This study was carried out to investigate the correlations of the sperm DNA fragmentation index (DFI) with semen parameters and apoptosis, and to investigate the effects of density-gradient centrifugation (DGC) and magnetic-activated cell sorting (MACS) on reducing the proportion of sperm with DNA fragmentation and protamine deficiency.

Methods: Semen analysis and a sperm DNA fragmentation assay were performed to assess the correlations between semen parameters and the DFl in 458 semen samples. Sperm with progressive motility or non-apoptosis were isolated by DGC or MACS, respectively, in 29 normozoospermic semen samples. The effects of DGC or MACS alone and of DGC and MACS combined on reducing the amount of sperm in the sample with DNA fragmentation and protamine deficiency were investigated.

Results: The sperm DFI showed a significant correlation $(r=-0.347, p<0.001)$ with sperm motility and morphology $(r=-0.114, p<0.05)$ but not with other semen parameters. The DFI $(11.5 \% \pm 2.0 \%)$ of semen samples was significantly reduced by DGC $(8.1 \% \pm 4.1 \%)$ or MACS alone $(7.4 \% \pm 3.9 \%)(p<0.05)$. The DFI was significantly further reduced by a combination of DGC and MACS $(4.1 \% \pm 1.3 \%, p<0.05)$. Moreover, the combination of DGC and MACS $(1.6 \% \pm 1.1 \%, p<0.05)$ significantly reduced the protamine deficiency rate of semen samples compared to DGC (4.4\% $\pm 3.2 \%)$ or MACS alone (3.4\% $\pm 2.2 \%)$.

Conclusion: The combination of DGC and MACS may be an effective method to isolate high-quality sperm with progressive motility, nonapoptosis, high DNA integrity, and low protamine deficiency in clinical use.

Keywords: Apoptosis; Centrifugation, Density gradient; Magnetic-activated cell sorting; Protamine deficiency; Sperm DNA fragmentation index

\section{Introduction}

It has been reported that DNA fragmentation may be caused by an exposure to high levels of seminal reactive oxygen species (ROS), failure of the antioxidant defense system $[1,2]$, or apoptosis $[1,3,4]$.

Received: Sep 6, 2016 · Revised: Sep 27, 2016 • Accepted: Oct 13, 2016 Corresponding author: Hee-Jun Chi

IVF Center, Mamapapa and Baby Clinic, 16F 247 Samsan-ro, Nam-gu, Ulsan 44707 , Korea

Tel:+82-52-258-6006 Fax:+82-52-258-6026 E-mail: hanna129@hanmail.net

This is an Open Access article distributed under the terms of the Creative Commons Attribution Non-Commercial License (http://creativecommons.org/licenses/by-nc/4.0/) which permits unrestricted non-commercial use, distribution, and reproduction in any medium, provided the original work is properly cited.
Sperm DNA damage by ROS is associated with chromatin packaging deficiencies and apoptosis. Protamine is an important molecule involved in packaging sperm DNA to protect sperm DNA from sources of external damage, such as ROS. Therefore, the percentage of sperm with a protamine deficiency has been found to show a positive correlation with the sperm DNA fragmentation rate [5]. Additionally, high ROS levels cause a decrease in the mitochondrial membrane potential, which is an initiating event in the apoptosis cascade [6].

Sperm DNA fragmentation is recognized as a major paternal factor that contributes to the failure of assisted reproductive technology (ART) programs [7], low fertilization rates [8,9], embryo cleavage [2], implantation [10], and pregnancy rates [11,12]. Although a sperma- 
tozoon with impaired DNA and normal morphology can fertilize an oocyte, the fertilized oocyte does not successfully proceed through further embryonic development [13], which indicates that the intact paternal genome is required for subsequent development of the embryo [14]. This conclusion is supported by the finding that the percentage of fragmented DNA was positively associated with recurrent pregnancy loss [15] and the miscarriage rate [16].

Several studies have found that the sperm DNA fragmentation index (DFI) had a significant correlation with sperm concentration [5,17], morphology [5,18,19], and motility [19-21]. However, controversy remains regarding the correlations between the DFI and semen parameters because some authors did not observe significant correlations between the sperm DFI and morphology [22], concentration [18,21], or motility [18].

It is generally accepted that the cut-off value of the sperm DFI should be $\geq 30 \%$, because this cut-off value has been reported to be positively correlated with lower rates of pregnancy and delivery $[17,23]$. To select sperm with normal DNA integrity (DFI $<30 \%$ ), intracytoplasmic morphologically selected sperm injection [24], hyaluronic acid-bound sperm selection [25,26], and the electrophoresis separation technique [27] are currently under study. Recently, new methods to isolate sperm with high DNA integrity using density-gradient centrifugation (DGC) $[28,29]$ and magnetic-activated cell sorting (MACS) have been introduced [30]. DGC significantly reduced the sperm DFI in the semen of intracytoplasmic sperm injection patients [31] and in teratozoospermic patients [32]. MACS not only efficiently reduces the sperm DFI [33], but also effectively separates apoptotic from non-apoptotic spermatozoa [34]. Moreover, a combination of DGC with conventional swim-up [35] or with MACS [34] may be a more effective way to isolate high-quality sperm with better motility and morphology, as well as a lower DFI. Tavalaee et al. [36] suggested that MACS before DGC is more useful for clinical sperm selection than MACS after DGC. However, MACS has limitations regarding sperm concentration and volume for loading due to the small size and volume of the column. Therefore, loading raw semen into the MACS column may reduce the filtering function of MACS and impede its ability to isolate motile non-apoptotic sperm, because dead/ apoptotic sperm bind to the MACS column in competition with motile/non-apoptotic sperm.

The objective of this study was to investigate the correlations between the sperm DFI and semen parameters and to establish an efficient method to isolate sperm with high DNA integrity and a low protamine deficiency rate using DGC and MACS.

\section{Methods}

This was a prospective study approved by the Institutional Review
Board (IRB) of Mamapapa \& Baby Clinic (Mamapapa IRB 2014-02). This study was carried out from December 2014 to November 2015.

\section{Semen samples and semen analysis}

Semen analysis and a sperm DNA fragmentation assay were carried out in 458 semen samples with the patients' approval. Semen samples were collected by masturbation after 3 to 7 days of sexual abstinence. After liquefaction of the semen at room temperature, semen analysis was performed according to World Health Organization (WHO) guidelines (2010) with computer-assisted semen analysis.

\section{Sperm DNA fragmentation assay}

Sperm DNA fragmentation was evaluated by the sperm chromatin dispersion test using the Halosperm kit (Halotech DNA, Madrid, Spain). Briefly, semen samples were diluted with phosphate-buffered saline to a concentration of $5-10 \times 10^{6} / \mathrm{mL}$, and $20 \mu \mathrm{L}$ was added to melted agarose and evenly mixed. Then a $20-\mu \mathrm{L}$ aliquot of the cell/ agarose suspension was placed on a pre-coated agarose slide and covered with a glass coverslip $(22 \times 22 \mathrm{~mm})$. The slides were allowed to solidify for 5 minutes at $4^{\circ} \mathrm{C}$, and then the coverslip was gently removed and the slide was immersed horizontally in acid denaturant for 7 minutes at room temperature. Subsequently, the slide was incubated in lysis buffer at room temperature for 25 minutes. After 5 minutes of washing in distilled water, the slide was dehydrated in a graded ethanol series (70\%, 90\%, and 100\%) for 2 minutes each and subsequently air-dried. The dehydrated slide was stained with the Wright-Giemsa stain and observed under a bright field microscope for halos. Four different dispersion patterns based on halo size were observed: (1) sperm nuclei with a large halo, (2) sperm nuclei with a medium halo, (3) sperm nuclei with a very small halo, and (4) sperm nuclei without a halo. Sperm with a large or medium halo were considered to be normal or non-fragmented, and sperm with a small halo or no halo were considered to have significant DNA fragmentation. The DFI was calculated as the percentage of fragmented sperm cells in a semen sample based on the assessment of at least 200 sperm cells per slide.

\section{Density-gradient centrifugation}

Of the 458 semen samples, 29 normozoospermic semen samples were selected and each sample was divided into two aliquots. One aliquot was washed with Ham's F-10 medium (Gibco, Life Technologies, Grand Island, NY, USA) and another aliquot was treated with DGC using PureSperm (Nidacon, Gothenburg, Sweden). This discontinuous density gradient consisted of two (80\% and $40 \%)$ 1-mL layers of PureSperm, and $2 \mathrm{~mL}$ of semen was deposited on the $40 \%$ layer. The gradient was then centrifuged at $400 \mathrm{~g}$ for 15 minutes. After centrifugation, the seminal plasma supernatant was discarded, and 
the sperm pellet was washed with Ham's F-10 medium by centrifugation for an additional 5 minutes and resuspended.

\section{Magnetic-activated cell sorting}

Externalization of phosphatidylserine (PS) in the inner plasma membrane is one of the earliest signs of apoptosis [37]. The MACS ART annexin V reagent kit (Miltenyi Biotec, Auburn, CA, USA) was used to filter apoptotic sperm using a binding protein with high affinity for PS, annexin V, conjugated to microbeads [38]. Each washed sample that underwent DGC was subsequently divided into 2 aliquots. One aliquot was kept untouched as the pre-MACS control, while another aliquot was subjected to the MACS technique for the depletion of apoptotic spermatozoa. To perform MACS, briefly, we adjusted the concentration of sperm to $10-50 \times 10^{6} / \mathrm{mL}$ with the MACS ART binding buffer. The sperm suspension was centrifuged at $300 \mathrm{~g}$ for 4 minutes, and then the pellet was resuspended with $100 \mu \mathrm{L}$ of the MACS ART Annexin V-conjugated microbead reagent. MACS binding buffer was added, making a final volume of $500 \mu \mathrm{L}$, and then mixed well and incubated for 15 minutes at room temperature with agitation. After incubation, the sperm suspension was loaded on a separation column, and the column was placed in a MACS multistand (Miltenyi Biotec) which had been previously rinsed with $1 \mathrm{~mL}$ of binding buffer. The sperm fraction retained in the column served as the annexin V-positive fraction (apoptotic sperm), due to binding to annexin V-conjugated microbeads, while the spermatozoa that passed through the column were annexin V-negative spermatozoa with an intact plasma membrane (non-apoptotic sperm).

\section{Sperm protamine deficiency assay}

Protamines are sperm-specific nuclear proteins that are essential

Table 1. Association of the sperm DFI with normal and abnormal semen parameters in 458 raw semen samples

\begin{tabular}{ll}
\hline Semen parameter (cut-off value) & Sperm DFI (\%) \\
\hline Volume $(1.5 \mathrm{~mL})$ & \\
$\quad$ Normal $(n=423)$ & $17.7 \pm 12.2$ \\
Hypospermic $(n=35)$ & $19.9 \pm 10.5$ \\
Concentration $\left(15 \times 10^{6} / \mathrm{mL}\right)$ & \\
$\quad$ Normal $(n=443)$ & $19.8 \pm 12.1$ \\
$\quad$ Oligozoospermic $(n=15)$ & $17.9 \pm 11.3$ \\
Motility $(40 \%)$ & $16.9 \pm 9.6$ \\
$\quad$ Normal $(n=272)$ & $23.9 \pm 14.4^{* *}$ \\
$\quad$ Asthenozoospermic $(n=186)$ & $17.1 \pm 11.4$ \\
Morphology $(4 \%)$ & $20.9 \pm 13.5^{*}$ \\
$\quad$ Normal $(n=293)$ & \\
Teratozoospermic $(n=165)$ & \\
\hline
\end{tabular}

DFI, DNA fragmentation index.

${ }^{*} p<0.05 ;{ }^{* *} p<0.001$. for the packaging of the condensed paternal genome in spermatozoa. Protamine deficiency is likely to be one of the contributing factors to DNA damage. The aniline blue assay is a representative test to assess sperm for protamine deficiency. Briefly, semen smears were prepared by spreading a drop of washed semen onto the glass slide and allowing it to air-dry. All smears were fixed in 3\% buffered glutaraldehyde for 30 minutes, stained with $5 \%$ aqueous aniline blue, and mixed with $4 \%$ acetic acid for 5 minutes. Spermatozoa were observed under oil immersion with bright-field microscopy at $1,000 \times$ magnification. The following staining patterns were observed: dark blue or partial blue staining of the sperm head (abnormal, protamine deficiency) and no staining (normal). The protamine deficiency rate was calculated as the percentage of the abnormal sperm cells in a semen sample based on the assessment of 200 sperm cells per slide.

\section{Statistics}

Results were expressed as mean \pm standard deviation. The Student's $t$-test was used to compare the DFI between semen samples with normal and abnormal semen parameters. The Pearson correlation coefficient was used to assess the relationships between the sperm DFI and semen parameters. The sperm DFI and protamine deficiency rates of semen samples treated with DGC or MACS alone and DGC with MACS were compared using analysis of variance, and the rates before and after treatment were evaluated using the paired $t$ test. Statistical analyses of the DFI and the protamine deficiency rate were performed after arcsine (square root) transformation. The $p$-values $<0.05$ were considered to indicate statistical significance. Statistical analysis was performed using SPSS ver. 11.0 (SPSS Inc., Chicago, IL, USA).

\section{Results}

We carried out semen analysis and a Halosperm assay in 458 semen samples to investigate the association of the sperm DFI with normal and abnormal semen parameters (Table 1). No differences were found in the DFI between the semen samples with normal or abnormal concentration and volume. However, the DFI of the semen

Table 2. Correlation between semen parameters and the sperm DFI in 458 raw semen samples

\begin{tabular}{lc}
\hline Semen parameter & Correlation between semen parameters and the DFI \\
\hline Concentration & $r=0.112$ \\
Motility & $r=-0.347^{* *}$ \\
Morphology & $r=-0.114^{*}$ \\
Volume & $r=0.089$
\end{tabular}

DFI, DNA fragmentation index.

${ }^{*} p<0.05 ;{ }^{* *} p<0.001$. 
samples with normal motility $(16.9 \% \pm 9.6 \%)$ was significantly lower than that of the asthenozoospermic semen samples $(23.9 \% \pm 14.4 \%$, $p<0.001)$. In addition, the DFI of teratozoospermic semen samples $(20.9 \% \pm 13.5 \%, p<0.05)$ was significantly higher than that of the semen samples with normal morphology $(17.1 \% \pm 11.4 \%)$.

The correlation coefficients between the sperm DFI and the semen parameters of whole semen samples were assessed (Table 2). Although sperm DFI showed a significant inverse correlations with sperm motility $(r=-0.347, p<0.001)$ or morphology $(r=-0.114, p<0.05)$, no significant correlation was found between the DFI and concentration $(r=0.112)$ and volume $(r=0.089)$.

The experimental process of the present study was illustrated as a diagram (Figure 1). Semen analysis and a sperm DNA fragmentation assay were carried out in 458 semen samples. Of the 458 semen samples, 29 normozoospermic semen samples were selected and each sample was divided into 2 aliquots. One aliquot was washed with medium and another aliquot was treated with DGC. The semen aliquots were subjected to the MACS for the separation into nonapoptotic and apoptotic sperm, and then sperm DFI and protamine deficiency were assessed.

The DFls in non-apoptotic and apoptotic sperm in washed semen samples and those that underwent DGC were compared (Figure 2). The DFI of non-apoptotic sperm was significantly lower than the DFI of apoptotic sperm in both washed $(7.4 \% \pm 3.9 \%$ vs. $17.2 \% \pm 3.7 \%$,

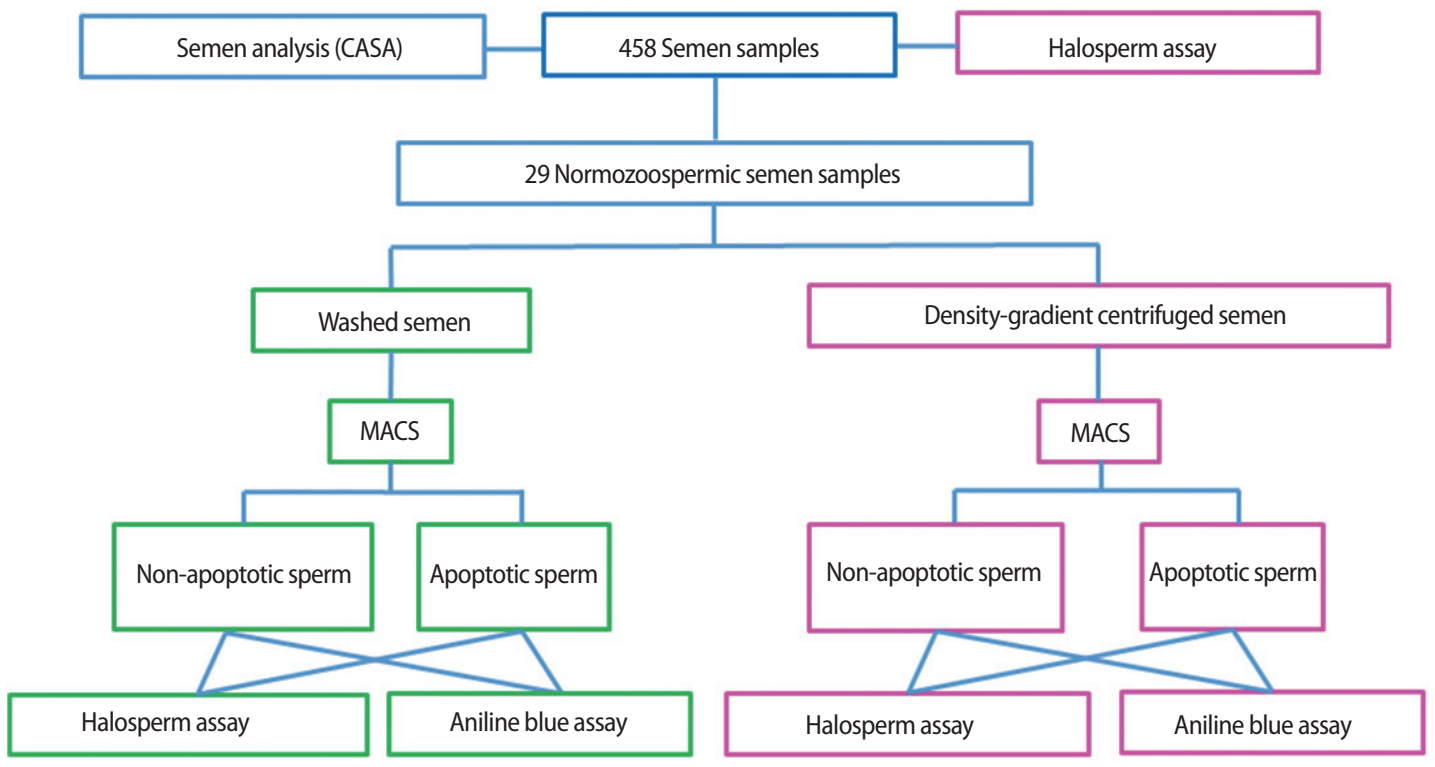

Figure 1. Diagram of experimental procedure. CASA, computer-assisted semen analysis; MACS, magnetic-activated cell sorting.

Sperm DNA fragmentation index (DFI \%)

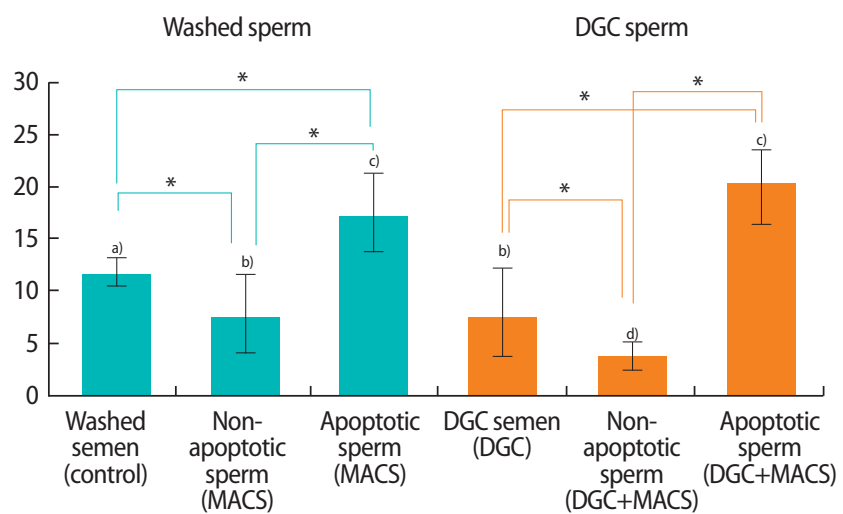

Figure 2. DNA fragmentation index of non-apoptotic and apoptotic sperm in washed and density gradient centrifuged (DGC) groups in 29 normozoospermic semen samples. MACS, magnetic-activated cell sorting. ${ }^{\text {a-d }}$ Results with different superscript alphabets are significantly different $\left({ }^{*} p<0.05\right)$.
Protamine deficiency rate (\%)

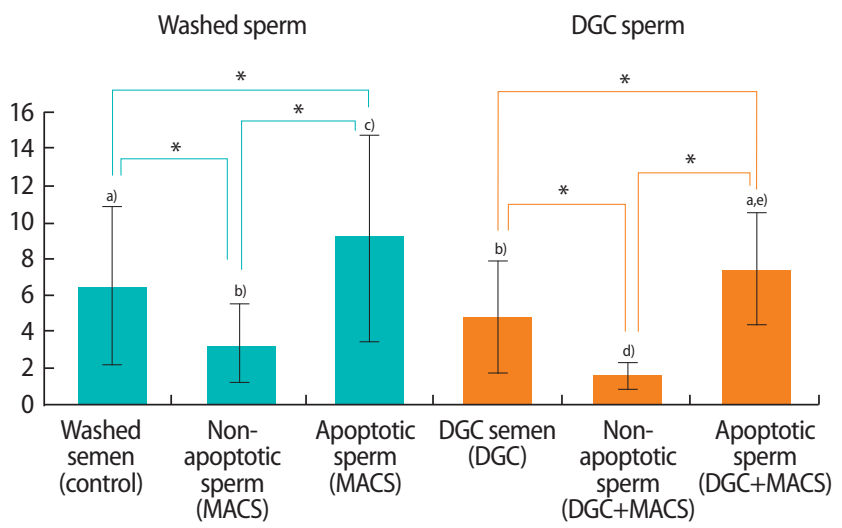

Figure 3. Protamine deficiency rates of non-apoptotic and apoptotic sperm in washed and density gradient centrifuged (DGC) groups in 29 normozoospermic semen samples. MACS, magnetic-activated cell sorting. ${ }^{a-e)}$ Results with different superscript alphabets are significantly different $\left({ }^{*} p<0.05\right)$. 
$p<0.05)$ and DGC semen samples $(4.1 \% \pm 1.3 \%$ vs. $20.2 \% \pm 4.0 \%$, $p<0.05)$. The DFI of washed sperm $(11.5 \% \pm 2.0 \%)$ was significantly reduced after treatment with DGC $(8.1 \% \pm 4.1 \%, p<0.05)$. The DFIs in the semen samples treated with MACS alone $(7.4 \% \pm 3.9 \%)$ or DGC alone $(8.1 \% \pm 4.1 \%)$ were significantly greater than the DFI in the semen samples treated with a combination of DGC and MACS $(4.1 \% \pm$ $1.3 \%, p<0.05)$.

Protamine deficiency rates between non-apoptotic and apoptotic sperm in washed semen samples and those that underwent DGC were compared (Figure 3). The protamine deficiency rates of nonapoptotic sperm in washed and DGC semen samples were $3.4 \% \pm$ $2.2 \%$ and $1.6 \% \pm 1.1 \%$, respectively, which were significantly lower than the rates in apoptotic sperm $(9.4 \% \pm 5.6 \%$ and $7.2 \% \pm 3.7 \%$, respectively, $p<0.05)$. The protamine deficiency rate in washed sperm $(6.4 \% \pm 4.2 \%)$ was significantly reduced after treatment with DGC $(4.4 \% \pm 3.2 \%, p<0.05)$. The protamine deficiency rates of the sperm samples treated with MACS alone $(3.4 \% \pm 2.2 \%)$ or DGC alone $(4.4 \% \pm 3.2 \%)$ were significantly higher than the rates of the semen samples treated with a combination of DGC and MACS $(1.6 \% \pm 1.1 \%$, $p<0.05)$.

\section{Discussion}

In previous studies, Trisini et al. [17] and Manochantr et al. [5] observed negative correlations between the sperm DFI and sperm concentration ( $r=-0.16$ and $r=-0.31$, respectively, $p<0.05$ ) and motility $(r=-0.17$ and $r=-0.46$, respectively; $p<0.05)$. Although we also observed a negative correlation between the DFI and motility $(r=-0.347, p<0.05)$, we did not observe a significant correlation between sperm concentration and the DFI $(r=0.112)$. The discrepancy in the results between the previous studies and the present study may be due to the difference in sexual abstinence period. Trisini et al. [17] collected semen after 2 days of abstinence, and Manochantr et al. [5] collected after 3 days of abstinence, while we collected after a comparatively long abstinence period (3-7 days). If the sexual abstinence period is prolonged, the sperm concentration is increased, while sperm motility and DFI will be decreased due to the increase in dead sperm.

Discrepancies exist in the correlation between the DFI and sperm morphology. In the present study, a significant correlation between sperm DFI and morphology was found, and some authors also have observed the correlation $[5,18,19,21]$, while Boushaba and Belaaloui [22] have not. This difference might be caused by differences in the cut-off values for morphology. In the present study, we used the cutoff value of $4 \%$ according to the fifth edition (2010) of the WHO guidelines, but Brahem et al. [18] used the cut-off value of $15 \%$ according to the fourth edition of the WHO guidelines (1999). Mano- chantr et al. [5] used a cut-off of 30\%, Sivanarayana et al. [19] used a cut-off of 14\%, and Boushaba and Belaaloui [22] used a cut-off of $30 \%$. Therefore, further study is needed to clarify how the choice of a cut-off value may affect the correlation between sperm morphology and the DFI.

We observed that sperm motility showed a significantly higher correlation with the DFI than other semen parameters. Similarly, a significant inverse correlation between sperm motility and DFI has been reported in many previous studies [5,17,19-22]. Therefore, no real debate exists regarding the finding that sperm motility has a significant correlation with the DFI. However, Brahem et al. [18] did not observe a significant correlation between the DFI and sperm motility ( $r=$ 0.051); nevertheless, they observed a significant decrease in the DFI (approximately $17.0 \%, p<0.01$ ) following DGC when compared with raw semen (32.8\%). In general, DGC is effective in isolating motile sperm from raw semen, resulting in a sharp increase of sperm motility [39]. Therefore, the results of Brahem et al. [18] suggest a close indirect association between the DFI and motility, although they did not observe a significant direct correlation between the DFI and motility. We also observed that the DFI of semen samples was significantly reduced by treatment with DGC (Figure 2). Morrell et al. [40] also reported that the sperm DFI $(20.9 \% \pm 8.1 \%)$ was significantly reduced by DGC $(12.8 \% \pm 8.1 \%, p<0.05)$. Therefore, the positive effect of DGC on reducing the DFI may be a reasonable result of the increased motility of the sperm sample due to the selection of motile sperm by DGC.

The DFI of the pre-MACS samples was significantly higher than that of the post-MACS samples (Figure 2). This result is similar to that of Degheidy et al. [41], who found that the sperm DFI was significantly reduced in post-MACS samples $(9.61 \% \pm 5.62 \%)$ compared with preMACS controls $(12.43 \% \pm 6.29 \%, p<0.05)$. We also observed that the DFI of non-apoptotic sperm $(7.4 \% \pm 3.9 \%, p<0.05)$ isolated with MACS was significantly lower than that of apoptotic sperm $(17.2 \%$ $\pm 3.7 \%$ ). This result is supported by previous reports that apoptosis is one of the main causes of sperm DNA fragmentation [1,3,4]. Although Degheidy et al. [41] reported that post-MACS samples showed no difference in sperm motility $(80.6 \% \pm 6.9 \%)$ compared with control samples $(80.9 \% \pm 7.7 \%)$, de Vantery Arrighi et al. [42] reported that the progressive sperm motility of post-MACS semen samples $(67.9 \% \pm 3.8 \%, p<0.05)$ was significantly better than preMACS control samples $(48.4 \% \pm 2.4 \%)$. Additionally, other studies have reported a significant inverse correlation between apoptosis and progressive motility [43-45]. Therefore, the positive effect of MACS on the isolation of sperm with high DNA integrity may result from the selection of non-apoptotic sperm with progressive motility. Moreover, we observed that the DFls of the semen samples treated with MACS alone $(7.4 \% \pm 3.9 \%)$ or DGC alone $(8.1 \% \pm 4.1 \%)$ were sig- 
nificantly higher than the DFI of the sperm samples treated with the combination of DGC and MACS $(4.1 \% \pm 1.3 \%, p<0.05)$. This synergistic effect of DGC and MACS has also been reported in previous studies [34,36]. Tavalaee et al. [36] reported that MACS before DGC was more useful for clinical sperm selection than MACS after DGC. Unlike their report, we suggest that MACS after DGC may be more effective, because the MACS column has a small volume $(0.5 \mathrm{~mL})$ and limitations in the sperm concentration $\left(10-50 \times 10^{6} / \mathrm{mL}\right)$ for loading. In some oligozoospermic or asthenozoospermic samples, loading of raw semen in the MACS column may result in the collection of insufficient sperm for subsequent DGC treatment. Moreover, the subsequent DGC treatment may result in an additional loss of sperm. We treated the raw semen with DGC in order to collect sufficient motile sperm and exclude immotile sperm and cell debris, and then subsequently treated the samples with MACS, which resulted in the collection of sufficient non-apoptotic sperm with progressive motility and high DNA integrity.

The protamine deficiency rates of non-apoptotic sperm were significantly lower than the rates of apoptotic sperm in both washed and DGC sperm samples (Figure 3), which suggests that a relationship exists between protamine deficiency and apoptosis. The protamine deficiency rates of the sperm samples treated with MACS alone $(3.4 \% \pm 2.2 \%)$ or DGC alone $(4.4 \% \pm 3.2 \%)$ were significantly reduced when the sperm samples were treated with the combination of DGC and MACS $(1.6 \% \pm 1.1 \%, p<0.05)$. This association showed a similar pattern to that of the sperm DFI with DGC and MACS, which suggests that protamine deficiency may be correlated with the DFI. This result is consistent with that of a previous study that reported a positive correlation $(r=0.68, p<0.01)$ between the percentages of protamine deficiency and sperm DNA fragmentation [5], which reflects the fact that protamine is involved in packaging sperm DNA to protect sperm DNA from sources of external damage. Therefore, our results confirm that protamine deficiency is a factor contributing to DNA damage.

In conclusion, treating sperm with a combination of DGC and MACS may be a useful method for isolating non-apoptotic sperm with motility, high DNA integrity, and stable chromatin packaging for clinical use.

\section{Conflict of interest}

No potential conflict of interest relevant to this article was reported.

\section{References}

1. Moustafa MH, Sharma RK, Thornton J, Mascha E, Abdel-Hafez MA, Thomas AJ Jr, et al. Relationship between ROS production, apoptosis and DNA denaturation in spermatozoa from patients examined for infertility. Hum Reprod 2004;19:129-38.

2. Seli E, Gardner DK, Schoolcraft WB, Moffatt O, Sakkas D. Extent of nuclear DNA damage in ejaculated spermatozoa impacts on blastocyst development after in vitro fertilization. Fertil Steril 2004;82:378-83.

3. Singh NP, Muller CH, Berger RE. Effects of age on DNA doublestrand breaks and apoptosis in human sperm. Fertil Steril 2003; 80:1420-30.

4. Wu GJ, Chang FW, Lee SS, Cheng YY, Chen CH, Chen IC. Apoptosis-related phenotype of ejaculated spermatozoa in patients with varicocele. Fertil Steril 2009;91:831-7.

5. Manochantr S, Chiamchanya C, Sobhon P. Relationship between chromatin condensation, DNA integrity and quality of ejaculated spermatozoa from infertile men. Andrologia 2012;44:187-99.

6. Wang X, Sharma RK, Sikka SC, Thomas AJ Jr, Falcone T, Agarwal A. Oxidative stress is associated with increased apoptosis leading to spermatozoa DNA damage in patients with male factor infertility. Fertil Steril 2003;80:531-5.

7. Smith R, Kaune H, Parodi D, Madariaga M, Rios R, Morales I, et al. Increased sperm DNA damage in patients with varicocele: relationship with seminal oxidative stress. Hum Reprod 2006;21: 986-93.

8. Virant-Klun I, Tomazevic T, Meden-Vrtovec H. Sperm singlestranded DNA, detected by acridine orange staining, reduces fertilization and quality of ICSI-derived embryos. J Assist Reprod Genet 2002;19:319-28.

9. Lewis $\mathrm{SE}$, Aitken RJ. DNA damage to spermatozoa has impacts on fertilization and pregnancy. Cell Tissue Res 2005;322:33-41.

10. Speyer BE, Pizzey AR, Ranieri M, Joshi R, Delhanty JD, Serhal P. Fall in implantation rates following ICSI with sperm with high DNA fragmentation. Hum Reprod 2010;25:1609-18.

11. Frydman N, Prisant N, Hesters L, Frydman R, Tachdjian G, CohenBacrie $\mathrm{P}$, et al. Adequate ovarian follicular status does not prevent the decrease in pregnancy rates associated with high sperm DNA fragmentation. Fertil Steril 2008;89:92-7.

12. Simon L, Brunborg G, Stevenson M, Lutton D, McManus J, Lewis SE. Clinical significance of sperm DNA damage in assisted reproduction outcome. Hum Reprod 2010;25:1594-608.

13. Avendano C, Franchi A, Duran H, Oehninger S. DNA fragmentation of normal spermatozoa negatively impacts embryo quality and intracytoplasmic sperm injection outcome. Fertil Steril 2010;94:549-57.

14. Sakkas D, Alvarez JG. Sperm DNA fragmentation: mechanisms of origin, impact on reproductive outcome, and analysis. Fertil Steril 2010;93:1027-36.

15. Zidi-Jrah I, Hajlaoui A, Mougou-Zerelli S, Kammoun M, Meniaoui I, Sallem A, et al. Relationship between sperm aneuploidy, 
sperm DNA integrity, chromatin packaging, traditional semen parameters, and recurrent pregnancy loss. Fertil Steril 2016;105: 58-64.

16. Agarwal A, Said TM. Role of sperm chromatin abnormalities and DNA damage in male infertility. Hum Reprod Update 2003;9: 331-45.

17. Trisini AT, Singh NP, Duty SM, Hauser R. Relationship between human semen parameters and deoxyribonucleic acid damage assessed by the neutral comet assay. Fertil Steril 2004;82:162332.

18. Brahem S, Mehdi M, Elghezal H, Saad A. Semen processing by density gradient centrifugation is useful in selecting sperm with higher double-strand DNA integrity. Andrologia 2011;43:196202.

19. Sivanarayana T, Krishna CR, Prakash GJ, Krishna KM, Madan K, Rani BS, et al. CASA derived human sperm abnormalities: correlation with chromatin packing and DNA fragmentation. J Assist Reprod Genet 2012;29:1327-34.

20. Varghese AC, Bragais FM, Mukhopadhyay D, Kundu S, Pal M, Bhattacharyya AK, et al. Human sperm DNA integrity in normal and abnormal semen samples and its correlation with sperm characteristics. Andrologia 2009;41:207-15.

21. Chi HJ, Chung DY, Choi SY, Kim JH, Kim GY, Lee JS, et al. Integrity of human sperm DNA assessed by the neutral comet assay and its relationship to semen parameters and clinical outcomes for the IVF-ET program. Clin Exp Reprod Med 2011;38:10-7.

22. Boushaba S, Belaaloui G. Sperm DNA fragmentation and standard semen parameters in algerian infertile male partners. World J Mens Health 2015;33:1-7.

23. Bungum $M$, Humaidan $P$, Axmon A, Spano M, Bungum L, Erenpreiss J, et al. Sperm DNA integrity assessment in prediction of assisted reproduction technology outcome. Hum Reprod 2007; 22:174-9.

24. Berkovitz A, Eltes F, Yaari S, Katz N, Barr I, Fishman A, et al. The morphological normalcy of the sperm nucleus and pregnancy rate of intracytoplasmic injection with morphologically selected sperm. Hum Reprod 2005;20:185-90.

25. Parmegiani L, Cognigni GE, Bernardi S, Troilo E, Ciampaglia W, Filicori M. "Physiologic ICSI": hyaluronic acid (HA) favors selection of spermatozoa without DNA fragmentation and with normal nucleus, resulting in improvement of embryo quality. Fertil Steril 2010;93:598-604.

26. Yagci A, Murk W, Stronk J, Huszar G. Spermatozoa bound to solid state hyaluronic acid show chromatin structure with high DNA chain integrity: an acridine orange fluorescence study. J Androl 2010;31:566-72.

27. Fleming SD, llad RS, Griffin AM, Wu Y, Ong KJ, Smith $\mathrm{HC}$, et al.
Prospective controlled trial of an electrophoretic method of sperm preparation for assisted reproduction: comparison with density gradient centrifugation. Hum Reprod 2008;23:2646-51.

28. Enciso M, Iglesias M, Galan I, Sarasa J, Gosalvez A, Gosalvez J. The ability of sperm selection techniques to remove single- or double-strand DNA damage. Asian J Androl 2011;13:764-8.

29. Rougier N, Uriondo H, Papier S, Checa MA, Sueldo C, Alvarez Sedo C. Changes in DNA fragmentation during sperm preparation for intracytoplasmic sperm injection over time. Fertil Steril 2013;100:69-74.

30. Said TM, Agarwal A, Grunewald S, Rasch M, Glander HJ, Paasch U. Evaluation of sperm recovery following annexin $\mathrm{V}$ magnetic-activated cell sorting separation. Reprod Biomed Online 2006;13: 336-9.

31. Wang M, Sun J, Wang L, Gao X, Lu X, Wu Z, et al. Assessment of density gradient centrifugation (DGC) and sperm chromatin dispersion (SCD) measurements in couples with male factor infertility undergoing ICSI. J Assist Reprod Genet 2014;31:1655-63.

32. Xue X, Wang WS, Shi JZ, Zhang SL, Zhao WQ, Shi WH, et al. Efficacy of swim-up versus density gradient centrifugation in improving sperm deformity rate and DNA fragmentation index in semen samples from teratozoospermic patients. J Assist Reprod Genet 2014;31:1161-6.

33. Bucar S, Goncalves A, Rocha E, Barros A, Sousa M, Sa R. DNA fragmentation in human sperm after magnetic-activated cell sorting. J Assist Reprod Genet 2015;32:147-54.

34. Lee TH, Liu CH, Shih YT, Tsao HM, Huang CC, Chen HH, et al. Magnetic-activated cell sorting for sperm preparation reduces spermatozoa with apoptotic markers and improves the acrosome reaction in couples with unexplained infertility. Hum Reprod 2010;25:839-46.

35. Grunewald S, Reinhardt M, Blumenauer V, Hmeidan AF, Glander $H J$, Paasch U. Effects of post-density gradient swim-up on apoptosis signalling in human spermatozoa. Andrologia 2010;42:12731.

36. Tavalaee M, Deemeh MR, Arbabian M, Nasr-Esfahani MH. Density gradient centrifugation before or after magnetic-activated cell sorting: which technique is more useful for clinical sperm selection? J Assist Reprod Genet 2012;29:31-8.

37. Lee SH, Meng XW, Flatten KS, Loegering DA, Kaufmann SH. Phosphatidylserine exposure during apoptosis reflects bidirectional trafficking between plasma membrane and cytoplasm. Cell Death Differ 2013;20:64-76.

38. Grunewald S, Paasch U. Sperm selection for ICSI using annexin V. Methods Mol Biol 2013;927:257-62.

39. Henkel RR, Schill WB. Sperm preparation for ART. Reprod Biol Endocrinol 2003;1:108. 
40. Morrell JM, Moffatt O, Sakkas D, Manicardi GC, Bizzaro D, Tomlinson $\mathrm{M}$, et al. Reduced senescence and retained nuclear DNA integrity in human spermatozoa prepared by density gradient centrifugation. J Assist Reprod Genet 2004;21:217-22.

41. Degheidy T, Abdelfattah H, Seif A, Albuz FK, Gazi S, Abbas S. Magnetic activated cell sorting: an effective method for reduction of sperm DNA fragmentation in varicocele men prior to assisted reproductive techniques. Andrologia 2015;47:892-6.

42. de Vantery Arrighi $C$, Lucas H, Chardonnens D, de Agostini A. Removal of spermatozoa with externalized phosphatidylserine from sperm preparation in human assisted medical procreation: effects on viability, motility and mitochondrial membrane potential. Reprod Biol Endocrinol 2009;7:1.
43. Chen Z, Hauser R, Trbovich AM, Shifren JL, Dorer DJ, Godfrey-Bailey $\mathrm{L}$, et al. The relationship between human semen characteristics and sperm apoptosis: a pilot study. J Androl 2006;27:112-20.

44. Zhang HB, Lu SM, Ma CY, Wang L, Li X, Chen ZJ. Early apoptotic changes in human spermatozoa and their relationships with conventional semen parameters and sperm DNA fragmentation. Asian J Androl 2008;10:227-35.

45. Sharbatoghli M, Valojerdi MR, Amanlou M, Khosravi F, Jafar-abadi MA. Relationship of sperm DNA fragmentation, apoptosis and dysfunction of mitochondrial membrane potential with semen parameters and ART outcome after intracytoplasmic sperm injection. Arch Gynecol Obstet 2012;286:1315-22. 